Journal of Mathematics and Statistics 4 (2): 94-97, 2008

ISSN 1549-3644

(C) 2008 Science Publications

\title{
New Approach to Compute Integral Transforms
}

\author{
A. Adawi and F. Awawdeh \\ Department of Mathematics, Hashemite University, \\ P.O. Box 150459, Zarqa13115, Zarqa, Jordan
}

\begin{abstract}
Integral transforms find special applicability within scientific and mathematical disciplines. A powerful and efficient homotopy methodology in evaluating integrals arises in integral transforms was presented. The method depends on solving a related first order linear differential equation by homotopy analysis method HAM. The Laplace transform, Fourier transform and the moment generating function of the standard normal distribution were used as test examples to show the efficiency of the method. The results obtained justify the advantage of this methodology. MATLAB 7 was used to carry out the computations.
\end{abstract}

Key words: Homotopy analysis method, integral transforms

\section{INTRODUCTION}

In mathematics, an integral transform is any transform $\mathrm{T}$ of the following form:

$$
(T f)(u)=\int_{t_{1}}^{t_{2}} K(t, u) f(t) d t
$$

The input of this transform is a function $f$ and the output is another function Tf. An integral transform is a particular kind of mathematical operator.

There are numerous useful integral transforms. Each is specified by a choice of the function $\mathrm{K}$ of two variables, the kernel function or nucleus of the transform. Some kernels have an associated inverse kernel $\mathrm{K}^{-1}(\mathrm{u}, \mathrm{t})$ which (roughly speaking) yields an inverse transform.

Mathematical notation aside, the motivation behind integral transforms is easy to understand. There are many classes of problems that are difficult to solve in their original representations. An integral transform maps an equation from its original domain (e.g., functions where time is the independent variable are said to be in the time domain) into another domain. Manipulating and solving the equation in the target domain is, ideally, much easier than manipulation and solution in the original domain. The solution is then mapped back to the original domain with the inverse of the integral transform.

As an example of an application of integral transforms, consider the Laplace transform and Fourier transform. The Laplace transform finds wide application in physics and particularly in electrical engineering, where the characteristic equations that describe the behavior of an electric circuit in the complex frequency domain correspond to linear combinations of exponentially damped, scaled and time-shifted sinusoids in the time domain. Other integral transforms find special applicability within other scientific and mathematical disciplines.

Sometime evaluating the integral in (1) is not easy. In this work an approximate method to evaluate such integrals will be introduced. The method depends on solving a related first order ordinary differential equation by the Homotopy Analysis Method $(\mathrm{HAM})^{[3-5]}$.

\section{MATERIALS AND METHODS}

Consider the initial value problem

$$
\begin{gathered}
y^{\prime}+p(t) y=f(t) \\
y(0)=0
\end{gathered}
$$

A possible way to solve the general first order linear equation (2a), is to multiply it by a suitable integrating factor $\mu(\mathrm{t})$ and thereby transform the equation into an integrable form. The simplest possible function for $\mu$, namely:

$$
\mu(\mathrm{t})=\exp \left(\int_{0}^{\mathrm{t}} \mathrm{p}(\tau) \mathrm{d} \tau\right)
$$

Corresponding Author: A. Adawi, Department of Mathematics, Hashemite University, P.O. Box 150459, Zarqa13115, Zarqa, Jordan 
Having found the function $\mu$, we obtain the solution of the initial value problem (2a-2b), HAM well addressed in $\mathrm{Liao}^{[3+5]}$ is employed. Consider:

$$
N[y(t)]=\frac{\partial y(t)}{\partial t}+p(t) y(t)-f(t)
$$

where, $\mathrm{N}$ is an operator, $\mathrm{y}(\mathrm{t})$ is unknown function and $\mathrm{t}$ the independent variable. Let $\mathrm{y}_{0}(\mathrm{t})$ denote an initial guess of the exact solution $y(t), h \neq 0$ an auxiliary parameter, $\mathrm{H}(\mathrm{t}) \neq 0$ an auxiliary function and $\mathrm{L}$ an auxiliary linear operator with the property $L[y(t)]=0$ when $\mathrm{y}(\mathrm{t})=0$. Then using $\mathrm{q} \in[0,1]$ as an embedding parameter, we construct such a homotopy:

$$
\begin{aligned}
& \hat{H}\left[\phi(t ; q) ; y_{0}(t), H(t), h, q\right]= \\
& (1-q) L\left[\phi(t ; q)-y_{0}(t)\right]-q h H(t) N[\phi(t ; q)]
\end{aligned}
$$

It should be emphasized that we have great freedom to choose the initial guess $\mathrm{y}_{0}(\mathrm{t})$, the auxiliary linear operator $\mathrm{L}$, the non-zero auxiliary parameter $\mathrm{h}$ and the auxiliary function $\mathrm{H}(\mathrm{t})$.

Enforcing the homotopy (3) to be zero, i.e.:

$$
\hat{\mathrm{H}}\left[\phi(t ; q) ; \mathrm{y}_{0}(\mathrm{t}), \mathrm{H}(\mathrm{t}), \mathrm{h}, \mathrm{q}\right]=0
$$

we have the so-called zero-order deformation equation:

$$
(1-q) \mathrm{L}\left[\phi(t ; q)-y_{0}(t)\right]=q h H(t) N[\phi(t ; q)]
$$

When $\mathrm{q}=0$, the zero-order deformation Eq. 4 becomes:

$$
\phi(\mathrm{t} ; 0)=\mathrm{y}_{0}(\mathrm{t})
$$

and when $\mathrm{q}=1$, since $\mathrm{h} \neq 0$ and $\mathrm{H}(\mathrm{t}) \neq 0$, the zero-order deformation Eq. 4 is equivalent to:

$$
\phi(t ; 1)=y(t)
$$

Thus, according to (5) and (6), as the embedding parameter $\mathrm{q}$ increases from 0 to $1, \phi(t ; q)$ varies continuously from the initial approximation $\mathrm{y}_{0}(\mathrm{t})$ to the exact solution $y(t)$. Such a kind of continuous variation is called deformation in homotopy.

By Taylor's theorem, $\phi(t ; q)$ can be expanded in a power series of $\mathrm{q}$ as follows:

$$
\phi(t ; q)=y_{0}(t)+\sum_{m=1}^{\infty} y_{m}(t) q^{m}
$$

where

$$
\mathrm{y}_{\mathrm{m}}(\mathrm{t})=\frac{1}{\mathrm{~m} !} \frac{\partial^{\mathrm{m}} \phi(\mathrm{t} ; \mathrm{q})}{\partial \mathrm{q}^{\mathrm{m}}} \mathrm{I}_{\mathrm{q}=0}
$$

If the initial guess $\mathrm{y}_{0}(\mathrm{t})$, the auxiliary linear parameter $\mathrm{L}$, the nonzero auxiliary parameter $\mathrm{h}$ and the auxiliary function $\mathrm{H}(\mathrm{t})$ are properly chosen so that the power series (7) of $\phi(t ; q)$ converges at $q=1$. Then, we have under these assumptions the solution series:

$$
y(t)=\phi(t ; 1)=y_{0}(t)+\sum_{m=1}^{\infty} y_{m}(t)
$$

For brevity, define the vector:

$$
\vec{y}_{n}(t)=\left\{y_{0}(t), y_{1}(t), y_{2}(t), \ldots, y_{n}(t)\right\}
$$

According to the definition (7), the governing equation of $y_{m}(t)$ can be derived from the zero-order deformation Eq. 4. Differentiating the zero-order deformation Eq. $4 \mathrm{~m}$ times with respective to $\mathrm{q}$ and then dividing by $\mathrm{m}$ ! and finally setting $\mathrm{q}=0$, we have the socalled $\mathrm{m}^{\text {th }}$-order deformation equation:

$$
\mathrm{L}\left[\mathrm{y}_{\mathrm{m}}(\mathrm{t})-\chi_{\mathrm{m}} \mathrm{y}_{\mathrm{m}-1}(\mathrm{t})\right]=\mathrm{hH}(\mathrm{t}) \Re_{\mathrm{m}}\left(\overrightarrow{\mathrm{y}}_{\mathrm{m}-1}(\mathrm{t})\right)
$$

where

$$
\Re_{\mathrm{m}}\left(\overrightarrow{\mathrm{y}}_{\mathrm{m}-1}(\mathrm{t})\right)=\frac{1}{(\mathrm{~m}-1) !} \frac{\partial^{\mathrm{m}-1} \mathrm{~N}[\phi(\mathrm{t} ; \mathrm{q})]}{\partial \mathrm{q}^{\mathrm{m}-1}}
$$

and

$$
\chi_{\mathrm{m}}= \begin{cases}0, & \mathrm{~m} \leq 1 \\ 1, & \mathrm{~m}>1\end{cases}
$$

Using $\mathrm{y}_{0}(\mathrm{t})=0, \mathrm{H}(\mathrm{t})=1$ and $\mathrm{L}[\phi(\mathrm{t} ; \mathrm{q})]=\frac{\partial \phi(\mathrm{t} ; \mathrm{q})}{\partial \mathrm{t}}$ with the property $\mathrm{L}(\mathrm{C})=0$, where $\mathrm{C}$ is integral constant. The $\mathrm{m}^{\text {th }}$-order deformation Eq. 11 for $\mathrm{m} \geq 1$ becomes:

$$
\begin{aligned}
y_{m}(t) & =\chi_{m} y_{m-1}(t)+h \int_{0}^{t}\left[y_{m-1}^{\prime}(\tau)\right. \\
& +p(\tau) y_{m-1}(\tau)-\left(1-\chi_{m} f(\tau)\right] d \tau
\end{aligned}
$$

When solving the first order DE:

$$
y^{\prime}+\frac{K_{t}(t, u)}{K(t, u)} y=f(t), \quad y(0)=0
$$


we have that:

$$
\mathrm{y}(\mathrm{t}) \mathrm{K}(\mathrm{t}, \mathrm{u})=\int_{0}^{\mathrm{t}} \mathrm{K}(\tau, \mathrm{u}) \mathrm{f}(\tau) \mathrm{d} \tau
$$

and so:

$$
\int_{t_{1}}^{t_{2}} K(t, u) f(t) d t=\lim _{t \rightarrow t_{2}^{-}} y(t) K(t, u)-\lim _{t \rightarrow t_{1}^{+}} y(t) K(t, u)
$$

\section{RESULTS AND DISCUSSION}

In this study, the Laplace transform, Fourier transform and the moment generating function of the standard normal distribution will be used as test examples to show the efficiency of the method (15).

Example 1: The Laplace transform of $f(t), t \geq 0$ is defined by ${ }^{[2]}$ :

$$
\ell[f(t)]=\int_{0}^{\infty} e^{-s t} f(t) d t
$$

and by (15), we get that:

$$
\ell[f(t)]=\lim _{t \rightarrow \infty}\left[e^{-s t} \sum_{m=1}^{\infty} y_{m}(t)\right]
$$

Let $f(t)=$ cost. Choosing $h=-1$, we have from (13) that:

$$
\begin{aligned}
y(t)= & \sum_{m=1}^{\infty} y_{m}(t)=(\sin t-s \cos t)\left(1-s^{2}+s^{4}-s^{6}+s^{8}-\cdots\right)+ \\
& \left(s-s^{3}+s^{5}-s^{7}-\cdots\right)\left(1+s t+\frac{1}{2} s^{2} t^{2}+\frac{1}{6} s^{3} t^{3}+\cdots\right) \\
= & \frac{1}{1+s^{2}} \sin t-\frac{s}{1+s^{2}} \cos t+\frac{s}{1+s^{2}} e^{s t}, \quad 0<s<1
\end{aligned}
$$

and when choosing

$$
\begin{aligned}
& \mathrm{h}=-\frac{1}{\mathrm{~S}}, \\
& y(t)=\sum_{m=1}^{\infty} y_{m}(t)=\operatorname{sint}\left[\frac{1}{s^{2}}-\frac{1}{s^{4}}+\frac{1}{s^{6}}-\frac{1}{s^{8}}+\cdots\right]- \\
& \cos t\left[\frac{1}{s}-\frac{1}{s^{3}}+\frac{1}{s^{5}}-\frac{1}{s^{7}}+\cdots\right]+ \\
& {\left[\frac{1}{\mathrm{~s}}-\frac{1}{\mathrm{~s}^{3}}+\frac{1}{\mathrm{~s}^{5}}-\frac{1}{\mathrm{~s}^{7}}+\cdots\right]\left[1+\mathrm{st}+\frac{1}{2} \mathrm{~s}^{2} \mathrm{t}^{2}+\frac{1}{6} \mathrm{~s}^{3} \mathrm{t}^{3}+\cdots\right]} \\
& =\frac{1}{1+\mathrm{s}^{2}} \sin \mathrm{t}-\frac{\mathrm{s}}{1+\mathrm{s}^{2}} \cos \mathrm{t}+\frac{\mathrm{s}}{1+\mathrm{s}^{2}} \mathrm{e}^{\mathrm{st}}, \quad|\mathrm{s}|>1
\end{aligned}
$$

In this line, we can obtain for $\mathrm{s}>0$ :

$$
\begin{aligned}
\ell[\cos t] & =\lim _{\mathrm{t} \rightarrow \infty}\left[\mathrm{e}^{-\mathrm{st}} \sum_{\mathrm{m}=1}^{\infty} \mathrm{y}_{\mathrm{m}}(\mathrm{t})\right] \\
= & \lim _{\mathrm{t} \rightarrow \infty}\left(\frac{1}{1+\mathrm{s}^{2}} \sin \mathrm{te}^{-\mathrm{st}}-\frac{\mathrm{s}}{1+\mathrm{s}^{2}} \cos \mathrm{te}^{-\mathrm{st}}+\frac{\mathrm{s}}{1+\mathrm{s}^{2}}\right) \\
= & \frac{\mathrm{s}}{1+\mathrm{s}^{2}}
\end{aligned}
$$

Example 2: Considering Eq. 15 as a definite integral over the range from $-\infty$ to $\infty$, then the right hand side of this equation defines the Fourier transform ${ }^{[2]}$ of $f(t)$. That is:

$$
\begin{aligned}
F[f(t)] & =\frac{1}{\sqrt{2 \pi}} \int_{-\infty}^{\infty} e^{i \omega t} f(t) d t \\
& =\frac{1}{\sqrt{2 \pi}}\left[\lim _{t \rightarrow \infty}\left(e^{i \omega t} \sum_{m=1}^{\infty} y_{m}(t)\right)-\lim _{t \rightarrow-\infty}\left(e^{i \omega t} \sum_{m=1}^{\infty} y_{m}(t)\right)\right]
\end{aligned}
$$

Let $f(t)=\sqrt{2 \pi} e^{-|t|}$ From (13), we have for $t>0$ :

$$
\begin{aligned}
y(t)= & \sum_{m=1}^{\infty} y_{m}(t)=-\left[1-\omega^{2}+\omega^{4}-\omega^{6}+\omega^{8}-\cdots\right] \\
& {\left[(1+i \omega)\left(1-t+\frac{1}{2} t^{2}-\frac{1}{6} t^{3}+\cdots\right)-\right.} \\
& \left.(1+i \omega)\left(1-i \omega t-\frac{1}{2} \omega^{2} t^{2}+\frac{1}{6} i \omega^{3} t^{3}+\cdots\right)\right] \\
= & -\frac{(1+i \omega)}{1+\omega^{2}}\left[e^{-t}-e^{-i \omega t}\right]
\end{aligned}
$$

and for $\mathrm{t}<0$ :

$$
\begin{aligned}
y(t)= & \sum_{m=1}^{\infty} y_{m}(t)=\left[1-\omega^{2}+\omega^{4}-\omega^{6}+\omega^{8}-\cdots\right] \\
& {\left[(1-i \omega)\left(1+t+\frac{1}{2} t^{2}+\frac{1}{6} t^{3}+\cdots\right)-\right.} \\
& \left.(1-i \omega)\left(1-i \omega t-\frac{1}{2} \omega^{2} t^{2}+\frac{1}{6} i \omega^{3} t^{3}+\cdots\right)\right] \\
= & \frac{(1-i \omega)}{1+\omega^{2}}\left[e^{t}-e^{-i \omega t}\right]
\end{aligned}
$$

We can conclude that:

$$
\begin{aligned}
& F\left[\sqrt{2 \pi} e^{-|t|}\right]=\lim _{t \rightarrow \infty}\left(e^{i \omega t} \sum_{m=1}^{\infty} y_{m}(t)\right)-\lim _{t \rightarrow-\infty}\left(e^{i \omega t} \sum_{m=1}^{\infty} y_{m}(t)\right) \\
= & \lim _{t \rightarrow \infty}\left(-\frac{(1+i \omega)}{1+\omega^{2}}\left[e^{-t} e^{i \omega t}-1\right]\right)-\lim _{t \rightarrow-\infty}\left(\frac{(1-i \omega)}{1+\omega^{2}}\left[e^{t} e^{i \omega t}-1\right]\right) \\
= & \frac{2}{1+\omega^{2}} .
\end{aligned}
$$


Example 3: Let $X$ be a continuous random variable with probability density function $\mathrm{f}(\mathrm{x})$ on $\mathrm{a}<\mathrm{x}<\mathrm{b}$ and zero elsewhere. The moment generating function of $\mathrm{X}$ is given by ${ }^{[1]}, M(t)=\int_{a}^{b} e^{t x} f(x) d x,-h<t<h$. To evaluate the moment generating function of a pdf and with the help of (15), we can use that:

$$
M(t)=\lim _{t \rightarrow b^{-}} e^{t x} y(x)-\lim _{t \rightarrow a^{+}} e^{t x} y(x)
$$

A random variable $\mathrm{X}$ that has a pdf of the form $\mathrm{f}(\mathrm{x})=\frac{1}{\sqrt{2 \pi}} \mathrm{e}^{-\frac{\mathrm{x}^{2}}{2}}, \quad-\infty<\mathrm{x}<\infty$ is said to have a standard normal distribution. We can find the moment generating function of the standard normal distribution as follows. By (13) we obtain:

$$
\begin{aligned}
y(t)= & \frac{\sqrt{2 \pi}}{2} e^{-t x} \operatorname{erf}\left(\frac{\sqrt{2}}{2} x-\frac{\sqrt{2}}{2} t\right)\left[1+\frac{1}{2} t^{2}+\frac{1}{8} t^{4}+\frac{1}{48} t^{6}+\cdots\right]+ \\
& \frac{\sqrt{2 \pi}}{2} e^{-t x} \operatorname{erf}\left(\frac{\sqrt{2}}{2} t\right)\left[1+\frac{1}{2} t^{2}+\frac{1}{8} t^{4}+\frac{1}{48} t^{6}+\cdots\right] \\
= & \frac{\sqrt{2 \pi}}{2} e^{-t x} \operatorname{erf}\left(\frac{\sqrt{2}}{2} x-\frac{\sqrt{2}}{2} t\right) e^{\frac{t^{2}}{2}}+\frac{\sqrt{2 \pi}}{2} e^{-t x} \operatorname{erf}\left(\frac{\sqrt{2}}{2} t\right) e^{\frac{t^{2}}{2}}
\end{aligned}
$$

In this line it is easy to verify that:

$$
\begin{gathered}
M(t)=\frac{1}{\sqrt{2 \pi}}\left[\lim _{t \rightarrow \infty} e^{t x} y(x)-\lim _{t \rightarrow-\infty} e^{t x} y(x)\right] \\
=\frac{1}{2}\left[\lim _{t \rightarrow \infty} \operatorname{erf}\left(\frac{\sqrt{2}}{2} x-\frac{\sqrt{2}}{2} t\right) e^{\frac{t^{2}}{2}}-\lim _{t \rightarrow-\infty} \operatorname{erf}\left(\frac{\sqrt{2}}{2} t\right) e^{\frac{t^{2}}{2}}\right] \\
=e^{\frac{t^{2}}{2}}, \quad-\infty<t<\infty
\end{gathered}
$$

\section{CONCLUSION}

The HAM was used for evaluating integrals arises in integral transforms. Three examples were discussed as demonstrations. It was concluded that the homotopy methodology is very powerful and efficient technique in evaluating a wide class of integral transforms and problems. It is also worth noting to point out that the advantage of the homotopy methodology is the fast convergence of the solutions.

\section{REFERENCES}

1. Hogg, R.V. and A. Craig, 1978. Introduction to Mathematical Statistics. 4th Edn. Mamillan Publishing Co. Inc., New York.

2. Kanwal, R.P., 1997. Linear Integral Equations Theory and Techniques. 2nd Edn. Birkhäuser Publisher, Boston, Cambridge.

3. Liao, S.J., 2003. An explicit analytic solution to the Thomas-Fermi equation. Applied Math. Comput., 144: 495-506.

4. Liao, S.J., 2003. Beyond Perturbation: Introduction to the Homotopy Analysis Method. 1st Edn. Chapman and Hall/CRC Press Publisher, Boca Raton.

5. Liao, S.J., 2004. On the homotopy analysis method for nonlinear problems. Applied Math. Comput., 147: 499-513. 